\title{
MERGING GALAXIES AND BLACK HOLE EJECTIONS
}

\author{
M.J. Valtonen \\ Turku University Observatory, Finland
}

In mergers of galaxies their central black holes are accumulated together. We show that fewblack hole systems arise which decay through black hole collisions and black hole ejections. The ejection statistics are calculated and compared with two observed systems where ejections have been previously suggested: double radio sources and high redshift quasars near low redshift galaxies. In both cases certain aspects of the associations are explained by the merger hypothesis.

The first stage in making of a few-black hole system is the formation of a supermassive black hole binary (Begelman, Blandford and Rees 1980). The binary forms when two galaxies merge and each contains a supermassive black hole in its center (Roos 1981, Gaskell 1985, Valtaoja, Valtonen and Byrd 1989). Such binaries are long lived, and if left undisturbed by other galaxy mergers, probably survive through the Hubble time.

But in many galaxies multiple mergers take place (Aarseth and Fall 1980, Mamon 1987) and thus three and possibly also four black hole systems come together. The instability of the few-body systems makes it difficult to collect large numbers of black holes in the same galactic nucleus. Only in some hierarchical systems can many black holes survive. Thus generally we have to consider what happens when three or four black holes, of comparable mass, come together.

As a representative example, let us take a few black hole system of the following parameters: diameter about 1 parsec, mass about $10^{9} \mathrm{M}_{\odot}$ and typical orbital speed of about $1000 \mathrm{~km} \mathrm{~s}^{-1}$. There is nothing unique about these parameters, and in particular the mass could be different by several orders of magnitude.

According to the usual orbital evolution of the few-body problem (see e.g. Szebehely and Peters 1967), close encounters between the black holes take place. Gravitational radiation predicted by General Relativity becomes important at these encounters, and sooner or later an encounter takes place where large amount of orbital energy is lost. Then either a tight, short lived binary forms or two black holes coalesce.

The formation of a tight binary can be disruptive to the system. Another black hole passing by the binary can pick up enough speed to leave the system or even the whole galaxy. This is most likely to happen when the average orbital speed of the binary is about $10000 \mathrm{~km} \mathrm{~s}^{-1}$.

For an escape from the galaxy, a four-body system is much more effective than a three-body system. After a tight binary has formed, a third black hole must find it rather soon. Otherwise the binary is destroyed by gravitational radiation before much else happens. In a four-body system the 
chaotic nature of orbits of the binary center of mass and the two other black holes more or less quarantees a strong interaction. In a three-body system a hierarchical situation often developes which leads to a stable binary and no escapes.

In a few-body system the closest encounters tend to take place between the heaviest bodies (e.g. Szebehely and Peters 1967). Therefore the three black holes involved in the ejection event are likely to be among the most massive ones in the system and the lightest one is left aside. When a supermassive black hole is ejected and escapes from a galaxy, the binary is also of comparable mass and recoils in the opposite direction. The binary soon coalesces and thus in essence the few black hole systems decay through ejecting pairs of black holes. The light fourth black hole remains at the center of the galaxy.

The ejected pairs are not exactly symmetric since the masses of the escaping black holes are generally unequal. However, there is a tendency, enforced by several factors in the dynamical process, to look for maximal symmetry in the ejected masses. When the distribution of mass ratios of the escaping bodies in many ejections is formed, it peaks strongly towards equal masses (Valtonen and Mikkola 1990).

Another kind of asymmetry may appear with respect to the directions of escape. There is some scattering of the escaping black holes from the fourth black hole which causes a small bending angle between the two lines of escape. In case of strong scattering also the escape speed is significantly affected, and thus unusually strong escape speed asymmetry should be associated with strongly bent lines of ejection.

Double radio sources form an obvious application of the symmetric pair ejection of supermassive black holes. In double radio sources filaments frequently connect two strong regions of radio emission with the nucleus of the central galaxy. They seem to show that whatever produces the radio emission in the outer radio lobes has come from the nucleus of the galaxy. The outer lobes themselves often contain a sharp very high surface brightness spot (Carilli, Perley and Dreher 1988, Laing 1989), apparently the primary source of energy. It could well be a supermassive black hole. The black hole may interact with the interstellar medium via a twin-exhaust beam of relativistic plasma (Blandford and Rees 1974), the outward side of which terminates at a shock front, a secondary hot spot (Valtaoja 1984).

In this view there would generally be three supermassive black holes in a given double radio galaxy, each associated with its own twin-exhaust beam (Figure 1). Unlike in the so called "unified schemes" (Orr and Browne 1982), the central beam would not generally point toward the outer components. This appears to be also the current observational situation (Pearson and Readhead 1988). Nor would there be any strong reason why the beams in the outer lobes should be aligned with each other or with the central beam. Of course, the various manifestations of the black hole 
trails would still line up with the outer lobes as long as there are no strong non-radial winds in the tenuous interstellar medium surrounding the galaxy.

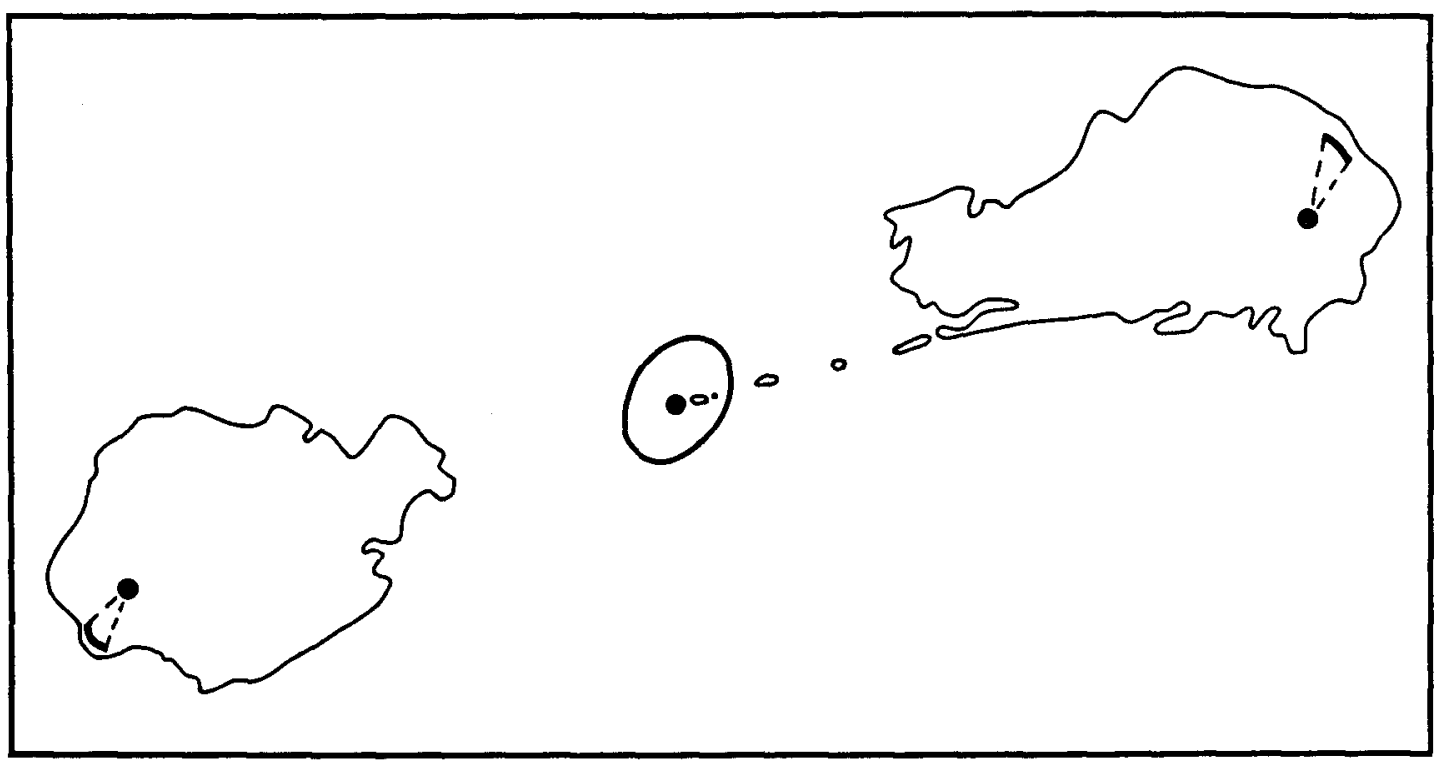

Fig. 1. Cygnus A and the three supermassive black holes (dots) according to the black hole ejection model. An ellipse describes the optical outline of the central galaxy, and the contour lines mark the regions of primary radio emission. They include a faint trail which connecis the center of the galaxy with one of the radio lobes. A slightly fainter trail is observed on the opposite side. Two of the jets in the model are indicated by dashed lines. The illustration is adapted from the work of Perley, Dreher and Cowan (1984).

This theory can be tested quantitatively by studying the relation of the central component in radio galaxies to the symmetry properties of the lobes. A bright and presumably massive central component should induce asymmetry via scattering the escaping black holes. This asymmetry should appear both as bending and as component distance asymmetry. Indeed, a strong correlation exists between the two kinds of asymmetry (Macklin 1981) and between the strength of the central component and asymmetry (Valtonen 1977).

In conclusion, the double radio sources are well explained by the black hole ejection model. Quantitative comparisons between the calculated models and observed double radio sources show excellent agreement, especially the connection between the central component and the outer lobes (Mikkola and Valtonen 1990). The alignment properties which have caused many problems in the so called unified schemes obtain a natural explanation in this theory.

Even though the few black hole process produces naturally ejections of rather symmetric pairs, there is also another decay mode where a relatively light black hole escapes from the galaxy without any significant opposite recoil. Single black hole ejections could be much faster than the pair ejections: ejection speeds exceeding $100000 \mathrm{~km} \mathrm{~s}^{-1}$ are possible. 
For example, we could consider the following process. Let the central supermassive black hole be surrounded by several smaller satellite black holes. Let the primary black hole mass be $10^{9} \mathrm{M}_{\odot}$ and let it have satellites of mass $10^{4} \mathrm{M}_{\odot}$ The system is stable against collapse caused by gravitational radiation for about one Galactic year $\left(\sim 2 \cdot 10^{8} \mathrm{yr}\right)$ if the satellite orbits are circular and the typical satellite speed is about $25000 \mathrm{~km} \mathrm{~s}^{-1}$. In that period of time a merger of galaxies may take place and bring a pair of massive black holes to the system. During the process of merger between the primary black hole and one of the newcomers, very high orbital speeds are obtained. Some fraction of the satellites will interact strongly with the high speed binary and will be ejected. This fraction is close to $100 \%$ when the binary speed is $50000 \mathrm{~km} \mathrm{~s}^{-1}$, and about $1 \%$ when the binary speed is $100000 \mathrm{~km} \mathrm{~s}^{-1}$.

Escape speeds are typically between one quarter and one third of the mean binary orbital speed, with the tail end of the distribution extending up to and even beyond the orbital speed. Thus we expect that satellites will be ejected with a speed distribution which extends all the way from $10000 \mathrm{~km} \mathrm{~s}^{-1}$ to $100000 \mathrm{~km} \mathrm{~s}^{-1}$, such that most of the ejections take place at the lower end of the distribution. An ejection of Markarian 205 from NGC 4319, as proposed by Sulentic and Arp $(1987 \mathrm{a}, \mathrm{b})$ could be well accommodated within this scenario.

Even if the supermassive black holes are not surrounded by satellite black holes, they should have a cluster of stars around them. The arrival of a pair of supermassive black holes would be equally disruptive to this system: stars and gas clouds (if stars are tidal disrupted, Rees 1988) would be ejected at speeds which extend up to the relativistic regime. 


\section{References}

Aarseth, S.J. and Fall, S.M. 1980, Ap. J. 236, 43

Begelman, M.C., Blandford, R.D. and Rees, M.J. 1980, Nature 287, 307

Blandford, R.D. and Rees, M.J. 1974, M.N.R.A.S. 169, 395

Carilli, C.L., Perley, R.A. and Dreher, J.H. 1988, Ap. J. (Lett.) 334, L73

Gaskell, C.M. 1985, Nature 315, 386

Laing, R.A. 1989, in Hot Spots in Extragalactic Radio Sources - Lecture Notes in Physics 327, ed. K. Meisenheimer and H.-J. Röser (Berlin: Springer), p. 27

Macklin, J.T. 1981, M.N.R.A.S. 196, 967

Mamon, G.A. 1987, Ap. J. 321, 622

Mikkola, S. and Valtonen, M.J. 1990, Ap. J. 348, in press

Orr, M.J.L. and Browne, I.W.A. 1982, M.N.R.A.S. 200, 1067

Pearson, T.J. and Readhead, A.C.S. 1988, Ap. J 328, 114

Perley, R.A., Dreher, J.W. and Cowan, J.J. 1984, Ap. J. (Lett.) 285, L35

Rees, M.J. 1988, Nature 333, 523

Roos, N. 1981, Astr. Ap. 104, 218

Sulentic, J.W. and Arp, H. 1987a, Ap. J, 319, 687

Sulentic, J.W. and Arp, H. 1987b, Ap. J. 319, 693

Szebehely, V. and Peters, C.F. 1967, A. J. 72, 876

Valtaoja, E. 1984, Astr. Ap. 140, 148

Valtaoja, L., Valtonen, M.J. and Byrd, G.G. 1989, Ap. J. 343, 47

Valtonen, M.J. 1977, Ap. J. 213, 356

Valtonen M.J. and Mikkola, S. 1990, in preparation 


\section{DISCUSSION}

Zasov: Can we expect any preferrable direction of black-hole ejection? Is it possible that the direction of the axis of rotation of a galaxy may be somehow distinguished?

Valtonen: There should not be any strong correlation between rotation and ejection axes.

Shlosman: I want to suggest the ultimate test for your ejection model. A SBH in the ISM or IGM would accrete at low M, produce an ion-supported torus and may create radio jets. Look for these jets centered on empty space.

Valtonen: Actually this may be happening inside the lobes of double radio sources, which one could interpret as the jet-IGM interaction regions. It appears that the jet originates from "empty" space outside the galaxy, from the point where the black hole has proceeded after the ejection. In some cases this point may be identified as a so-called $B$ hot spot.

Roos: I agree that many galaxies might harbour massive black holes. However, I think that a subsequent merger may trigger the flow of stars (and perhaps gas) through the orbit of the central binary. The central binary may then lose orbital energy as the third hole spirals inward and merge before the third hole reaches it. In that case ejections would not occur.

Valtonen: This process may be relevant to low-mass black-hole binaries, in which case it would set a lower limit (around $10^{7}$ $M_{O}$ ) to the black hole masses which are involved in ejection. But it is not certain that the process you mentioned operates in many galaxies since it requires a slow approach by the incoming body and practically circular approach orbit. We did not obtain such orbits in our simulations. 